\title{
The changing profile of the translator profession at the European Central Bank
}

\author{
Rossana Villani ${ }^{1}$ \\ ${ }^{1}$ Head of the Language Services Division, European Central Bank \\ Rossana.Villani@ecb.europa.eu
}

\begin{abstract}
The translator profile at the European Central Bank (ECB) has evolved increasingly rapidly over the past years. Staff of the linguistic services moved from their traditional set of tasks - translation, editing and proofreading, often done on print-ups or in plain Word documents - to dealing with a multitude of tasks, such as project management, procurement, recruitment and training, and lately cultural mediation. But the real game changer in this transformation has been the quantum leap that technology applied to translation (computer-assisted translation (CAT) and machine translation (MT)) has enabled in complex organisational structures. This brought about significant challenges in terms of people and change management, but also presented huge opportunities and the chance to broadcast the ECB's language services as a cutting-edge professional unit within the organisation. The transformation is still underway, but we are not in uncharted waters anymore. In fact, we are broadening our interest in new technologies to make our communication more accessible, even beyond the pure translation function.
\end{abstract}

\section{Introduction}

The European Central Bank is one of the institutions of the European Union. Founded in 1998, it is the central bank of a group of currently 19 countries in the EU which have adopted the euro as their common currency, the equivalent of the Federal Reserve System in the United States or the Bank of England in the United Kingdom.

The ECB's key tasks include keeping inflation under control, keeping the banking system sound and the issuance of euro banknotes and coins. The bank's headquarters are in Frankfurt am Main, Germany's financial capital.

The bank's linguistic area is called the Language Services Division. Somewhat paradoxically, it is the largest division in the bank in terms of staff; but at the same time, it is a relatively small operation compared to the linguistic services of other EU institutions.

The Language Services Division is part of the Communications department, which reflects the fact that for an international organisation such as the ECB, multilingual communication forms an integral part of its communication. The Division was incorporated into the Communications department as recently as 2013, which is indicative of some of the trends that will be addressed. 


\section{Twenty years of change}

\section{So how has our translator's world transformed over the past 20 years?}

Starting with target audiences, we have observed a strong shift of focus from expert to non-expert audiences.

This was also reflected in the broadening range of text types. New text types were also added with the emergence of new channels of communication, and the digital revolution in communications also broadened the range of file formats that translators are exposed to. On the resources front, the focus clearly shifted from investing in internal staff to outsourcing, which meant a higher coordination overhead for the function as a whole and more quality control for the translator. However, for the translator, the biggest change of all was to experience how technology slowly crept into and gradually took over their day-to-day work. In addition to being expected to use computer-assisted translation tools and (with the quantum leap in artificial intelligence) machine translation, they were suddenly exposed to a broad range of tools and technical environments to perform their translations (e.g. web editors, social media platforms), task management and administration systems, workload measurement systems etc. With management style becoming less top-down and more participative, translators were empowered to take on small coordination tasks or manage small projects (in dedicated task forces), which required training in a number of soft skills. The overall impact of all these factors was either a change in focus of translators' existing activities (e.g. translating new text types, post-editing instead of classic editing) or the need to learn to perform completely new activities like transcreation, cultural mediation and project management.

The factors driving this complex set of trends can be grouped and analysed in different ways, and what you see here is my personal take.

The first major factor is the digital revolution that has reshaped the communication landscape over the last few years. A series of technological inventions and breakthroughs have created new communication platforms as well as new ways of communicating - also multilingually. The speed of communication has increased exponentially, the tone has become lighter, the language simpler, and there is a flood of information at our fingertips, which requires new ways of processing.

Second, there is a set of factors which are specific to the ECB. The financial crisis of 2007-09, followed by the sovereign debt crisis in the euro area led to fundamental institutional innovations in the EU: as of November 2014, the ECB (together with the national authorities) was given the responsibility to supervise large banks operating in the euro area. As a result, in a matter of years, the organisation almost doubled in size, and support areas such as the language services were now supposed to serve a much larger organisation. The impact on the translation function was huge: new counterparts, new subject matters, new text types, a spike in demand (some of it cyclical, some of it completely unpredictable) etc. We were granted some additional resources, but this was not commensurate with the increase in workload.

Another part of the ECB's response was its decision to beef up the communication area both in terms of its policy communication as well as the need to keep up with the latest communication trends. For the translation function this meant the organisational 
(and functional) integration into the communication area. A new communications approach was adopted: communication was to become pro-active, focus more on nonexpert audiences and satisfy the increased demand for transparency and accountability. All of this had a direct impact on our day-to-day work in terms of text types, registers, tools, deadlines, volumes and visibility.

Finally, the translation industry has also undergone sweeping changes. Here are just a few key aspects: (i) again, technology plays a key role (CAT tools, advances in MT), (ii) a broader range of client needs need to be met (shorter deadlines, broad variety of text types and file formats), and (iii) there is a huge pressure to drive down costs (through the use of technology, scaling up operations and heavy outsourcing).

\section{A new vision}

How did we go about transforming ourselves into an agile, cutting-edge service that is better placed to weather the challenges in years to come?

First of all, we needed to invest in job coordination. For example, we reorganised our coordination assistant teams and created several smaller teams with specialised tasks (contract management, translation task coordination, project teams). In addition, we created "team lead" positions, filled mostly by former translators with a coordination profile who would bridge the functional gap between translators and management, relieving managers of work-coordination tasks and adding an organisational layer with a strong operational background benefitting the translators.

Second, we switched to a more inclusive style of management and involved translators in certain projects and tasks. In particular, we created a set of task forces which deal with specific operational and organisational issues.

Third, we switched to a more pro-active stakeholder management to make sure that we can influence our requesters early on in the production process.

We increased our outsourcing activity especially in the languages heavily involved in banking supervision work. Over the years, we have introduced various measures to make a sufficient capacity buffer available to us at any point in time.

These measures made perfect sense and have increased our efficiency enormously, however the real game changers were the ones which were related to technology simply because the common thread in the transformation that we were seeing was technology. Of the technology-related measures, three are key: investment in language technology, investment in business process development and adopting data-driven decision making.

\section{$4 \quad$ Language technology tools}

At the ECB, we have been using computer-assisted translation tools since 1998, namely Trados Studio and MultiTerm. In addition, we have a set of additional custom-made tools which automate certain processes to render them more efficient.

For example, special custom-made editors were developed by our webmasters which optimise the translation workflow of our website. These allow the translators to directly 
make changes on their language version of webpages before they are published. Given that our webmaster (naturally) do not speak all official EU languages, it makes this process much less error-prone.

Before the creation of these special text editors for website translation, the webmasters had to copy the translatable text from the html files into Microsoft Word which the translators translated and returned to the webmasters. The translations were then manually copied into the HTML files by the webmasters. Thereafter the translators proofread the pages and had to report the linguistic changes to the webmasters in Microsoft Word, who then in turn tried to implement these linguistic amendments on the individual language web pages. This was an extremely inefficient and very frustrating process for both, the translators and the webmasters, as it involved a lot back and forward, esp. for languages the webmasters didn't speak or let alone read (e.g. Greek). It was very time-consuming. The new workflow involves the translation of html files in Trados Studio, with the proof-reading and finalisation of the webpage being done in special editors. Thanks to the special editors which are now used by the translators the whole process has become much leaner and streamlined and allows everybody to focus on what they can do best: the webmasters focus on the design while the translators can focus on the linguistic content.

We have developed a tool which automates the creation of the Trados Studio projects and automatically applies all language specific settings to the individual projects. This saves considerable amounts of time and allows the translators to start working sooner.

We also use a set of tools and macros to deal with repetitive and time-consuming tasks. These are developed by a small team of language technologists (6 people) who have a background in computational linguistics and translation. They assist the linguists and their freelance collaborators in case of any technical issues with the tools and workflows and perform a business-critical help desk function for the translators. The language technologists also offer advice on the workflow of particular file formats in order to make the most efficient use of the tools.

Over the past years there have been many changes also in the language technology field which have had a huge impact on the translators' work.

We started with the Translator's Workbench in 1998 translating only Word files. Today, we can translate a large variety of file formats for different purposes, using many different tools. It is a little like changing from using a horse and cart to now driving a Formula 1 car: it is much faster and the output that can be achieved is much higher.

In addition to the obvious reasons for introducing computer-assistant translation tools in 1998, we had an additional one, namely that we only had one translator per language, translating out of English. We therefore saw the need to record all our translations in a database to have this available for consistency when the translator went on holiday and was replaced by a freelance on site. As of 2005 , we used an additional Trados application (TagEditor) which allowed the processing of more file formats.

As of 2008, we also had to translate more complex products, such as educational games which were translated in xml format, implying a big change for the translators.

In addition to the regular translation of Microsoft Office documents and html files, over the years we were increasingly asked to translate different products from different 
customers throughout the Bank. We needed to be agile and remain open to deal with different file formats.

For example, we were asked to translate websites that were hosted externally, such as the temporary external website that was set up in 2013 for the introduction of the new euro (Europa series) banknotes. The translators had to learn how to use a new content management system and work in that environment.

In the context of the new euro banknotes, we also translated educational games such as €uro Cash Academy, which was created to help people familiarise themselves with the banknotes' new security features. It still exists as an App.

The ECB has a very interesting Visitor Centre which unfortunately is temporarily closed due to the current restrictions but also exists as a virtual tour which you can access from the ECB's website. There are many digital interactive tools which had to be translated in different formats. For this project, the translators had to carefully consider the target audience and to adapt the style, register and terminology accordingly. You might like to have a look at it or come to see the Visitor Centre if you happen to be in Frankfurt once the pandemic restrictions are lifted.

\section{$5 \quad$ Machine Translation}

We cooperate with other EU institutions that run language services. For instance, we financially contribute to the running of the linguistic tools, including eTranslation (the machine translation tool developed by the European Commission).

Currently, eTranslation is used by two sets of users at the ECB - banking supervision experts and linguists. The banking supervision function produces a very high number of pages which must be translated in several language combinations. Given that the ECB's translation department is not staffed to handle these volumes of pages, it was agreed that the supervision experts would use eTranslation for gisting purposes and identify the parts of text for which they needed accurate human translation. Between 2014 and 2019, the supervisors were using the statistical machine translation engines of eTranslation (aka MT@EC). Since the rollout of the new neural engines (eTranslation), they have reported a considerable improvement in the translation quality.

Our linguists use machine translation in conjunction with the computer-assisted translation tools, as an additional resource which offers a translation if there is none in the translation memories. We consider this the most efficient way of using it, as it allows the best of both worlds given that you also receive the high-quality translations from the translation memories which were populated by professional linguists. The more technology is used, especially machine translation, the more it proves how critical the human component and added value is in the translation process. To arrive at the perfect translation with regard to style, message, register and terminology, the translators must always have the final 'word'. Together with the European Commission, we are currently creating a domain-specific engine with ECB documents as well as documents from the national central banks, which should yield even higher quality output. These engines will be ready by the end of August. 
Machine translation is here to stay. We must embrace it, face it head-on and use it to our advantage.

\section{Analytics}

The Analytics team in the Language Services Division has developed a reporting component which monitors the usage of the machine translation tool, combining two metrics: the number of pages and the number of requests. The data shows that there is a steadily increasing trend with a high degree of daily volatility ranging from 0 to over 30,000 (pages) and from 1 to over 1,000 (requests), depending on the submission of documents from commercial banks of the euro area which cannot be the same every month; this increase in machine translation usage is especially noticeable since the rollout of the neural engine.

Data is compiled by language pairs and also on the annual translation output, in terms of pages and requests. In most cases the monthly eTranslation output is higher than annual human translation output.

Other data is being compiled to provide us with more granular information on the use of the machine in order for us to prioritise the training of the engines.

\section{Translation workflow system}

For a long time, translation jobs were managed via emails: requests came in emails, they were assigned to the translators concerned in emails and the final product was delivered in emails. As workload increased and processes became more complex, this system became untenable but for many years no viable solution was found. In 2012 we finally launched a project to set up such a workflow, based on a well-established IT system in the bank, to provide a platform with which translators, assistants and managers interact and without which we simply could not function these days.

The main challenge for the translators was that they had to learn to use yet another digital tool and completely alter the way they were managing their day-to-day tasks. The key change management challenge was to raise translators' awareness of the need to invest time in learning how to use the tool and to use it correctly, in order to reap long-term benefits.

In any institution, if you want to persuade your decision-makers of the validity of your argument or business case, you have to learn to speak their language, which in our case is the language of numbers. In addition, investing in data analytics was a logical consequence of creating a task management tool. This tool is a rich depository of operational data waiting to be explored and used for resource-related decisions.

The key change management challenge here was to gain staff acceptance that data is being collected on what they were doing ("Big Brother is watching you"-effect). We had to make sure staff understands that numbers are not (ab)used out of context and need to be interpreted carefully. We managed to get this staff buy-in primarily through information sessions and by highlighting success stories (getting new resources, conflict management, better planning). 


\section{Conclusion}

In summary, there were several challenges which the translators have had to face and deal with over the past few years, notably: (i) the number of file formats involving different or specialised workflows; (ii) the increasing workload and handling different types of jobs simultaneously; (iii) tighter deadlines; (iv) an increased number of tasks (beyond pure translation ), e.g. having to learn how to use new tools; (v) the different target audiences and platforms, e.g. experts vs general public; and of course (vi) the reluctance to change, which is only human.

Most recently, the coronavirus pandemic and the forced working from home since March 2020 entailed significant additional changes for the translators. They were forced to deal with technical problems by themselves since they could not rely on an on-site help desk function. It was a very challenging time both professionally and personally and they also had to learn how to work on remote platforms, with new communication tools (WebEx, Microsoft Teams, Jabber, etc), with only virtual access to support teams.

Over the years we have had to address these challenges and fears that the translators were facing. The changes are happening faster and faster. Furthermore, today's technology is no longer just an option which we can decide to use or not, but it is an absolute must. The change management process had to be planned very carefully, especially with the arrival of machine translation which could have been perceived as a professional threat to the translators' jobs.

To support the technological changes, since 1998 we have been offering dedicated expert language technology support by our language technologists who have become a critical player in the entire translation process given the huge role that technology plays in our translation environment today.

In order to arrive at more acceptance of changes, we involved the translators when planning translation workflows or when developing our new tools. This has proven very helpful and effective as the tools are being devised 'around' the translators' needs as much as possible. This also helped in the acceptance of the workflows and tools by the translators as they felt involved and saw the benefits of the new tools as they were designed to better suit their needs.

To mitigate the translators' scepticisms of machine translation, we invited speakers from academia, European institutions, and the private sector to talk about the topic and reinforce the message that machine translation is 'everywhere' and is here to stay. The speakers outlined the limitations but also the opportunities which machine translation can offer them. How to use machine translation to their advantage and at the same time to use it as a possibility to affirm the professional value of the human translator. Moreover, we involved the translators in the assessment projects of machine translation output, thereby bringing them closer to realising that it might be of use to them. We did not want to leave anybody behind but encourage all translators to move forward and keep abreast of technology. Over the past few years, we have noticed that a change process is going on in the mindset of our translators with regard to machine translation. We perceive an increasing openness, acceptance, and willingness to embrace it, as they are exploring how they can use it to their advantage. 
To be able to handle the increased workflow, we have also been making increased use of outsourcing.

In addition to the training offered in the context of the language technology tools, and in order to prepare themselves better for all the different demands we are facing, translators have been attending training courses such as 'Writing for the Web' to better reach the dedicated target audience, 'Post-editing training' to better integrate machine translation output in the translation work and 'Subtitling training'.

To conclude, the translator's profile has changed dramatically and will continue to do so. The translator of the future certainly needs to be IT-savvy, detail-oriented and able to follow instructions, but at the same time be agile enough to switch between text types, be able to deal with multiple tasks quickly and be eager to learn new skills, both on the soft skills side and on the IT side. They will also need the ability to adapt quickly to unexpected situations and jump between micro and macro level tasks.

At the European Central Bank, we have come a long way and been successful in embracing the changes that these turbulent times have presented to us. However, this is a journey that has not yet ended and if there is one thing that we have learned: public organisations of the future cannot afford to lag behind when it comes to the fast pace of change in the world around them. 